\title{
High heat flux testing of mm-thick tungsten coatings on carbon-fiber composites for the JT-60SA tokamak
}

\author{
M Fukumoto ${ }^{1}$, H Greuner ${ }^{2}$, B Böswirth ${ }^{2}$, S Elgeti ${ }^{2}$, H Maier $^{2}$, R Neu ${ }^{2,3}$, T Nakano ${ }^{1}$, N \\ Kuroki $^{4}$, K Noumi $^{4}$, T Takabatake ${ }^{4}$, Yoshida $^{5}$
}

${ }^{1}$ National Institutes for Quantum and Radiation Science and Technology, 801-1 Mukoyama, Naka-shi, Ibaraki-ken 311-0193, Japan

${ }^{2}$ Max-Planck-Institut für Plasmaphysik, Boltzmannstrasse 2, 85748 Garching, Germany

3 Fakultät Maschinenwesen, Technische Universität München, Boltzmannstrasse 15, 85748 Garching, Germany

${ }^{4}$ Tocalo Co. Ltd, 14-3 Minamifutami, Futami-cho, Akasi-shi, Hyogo 674-0093, Japan

${ }^{5}$ Research Institute for Applied Mechanics, Kyushu University, 6-1 Kasuga-koen, Kasuga-shi, Fukuoka, 816-8580, Japan

E-mail: fukumoto.masakatsu@qst.go.jp

\begin{abstract}
High heat flux tests have been performed on $0.4 \mathrm{~mm}$ thick $\mathrm{W}$ coatings produced by vacuum plasma spraying on carbon-fiber composites (CFCs) for the future actively cooled $\mathrm{W}$-coated CFC divertor target in JT-60SA. The surface temperature of the $\mathrm{W}$ coating increased up to $2000{ }^{\circ} \mathrm{C}$ at $15 \mathrm{MW} / \mathrm{m}^{2}$ for $5 \mathrm{~s}$. No severe morphological changes, for example, melting, cracking and delamination were observed although recrystallization and grain growth were found in the $\mathrm{W}$ coating. Cyclic loading at $15 \mathrm{MW} / \mathrm{m}^{2}$ for $3 \mathrm{~s}$ with 50 cycles and additional 50 cycles with longer pulse length of $4 \mathrm{~s}$ produced again no severe morphological changes. Heat transfer analysis of the W-coated CFC sample during the high heat flux test showed that thermal resistance between $\mathrm{W}$ coatings and CFCs was negligibly low. This analysis also showed that the surface temperature of the $1 \mathrm{~mm}$ thick $\mathrm{W}$ coating on the actively cooled CFC monoblock divertor target in JT-60SA will be higher than the recrystallization temperature, $1200{ }^{\circ} \mathrm{C}$, at the steady state heat flux of $15 \mathrm{MW} / \mathrm{m}^{2}$. Therefore, a reduction of armor thickness of the future actively cooled W-coated CFC monoblock divertor target in JT-60SA could be necessary to decrease the surface temperature to less than the recrystallization temperature.
\end{abstract}




\section{Keywords}

Tungsten, Tungsten coating, Vacuum plasma spray, CFC, High heat flux, JT-60SA

\section{PACS numbers}

28.52.Fa, 28.52.Lf, 52.40.Hf, 81.15.Rs

\section{Introduction}

In super conducting tokamak JT-60SA, plasma productions shall be performed with carbon plasma facing components (PFCs) to complete the first mission of JT-60SA [1]. The first wall is covered by fine grain graphite (FGG) tiles. The divertor is a monoblock type using monoblock CFCs to remove heat fluxes of up to $15 \mathrm{MW} / \mathrm{m}^{2}$ which is the maximum heat flux expected on the divertor target in JT-60SA [2]. The others such as baffles and domes are covered by CFC tiles. After the first mission is completed, carbon PFCs are replaced by tungsten ones. The most likely method to cover the first wall is the use of $\mathrm{W}$ coatings on the FGG tiles. Because the W-coated FGG tiles on the first wall are replaceable every years by a remote handling manipulator and the expected erosion is low, thin $\mathrm{W}$ coatings will be acceptable. On the other hand, thick $W$ surviving for $\sim 5$ years is necessary on the inner and the outer divertor target because the divertor, which is a cassette type similar to ITER, is planned to be replaced every $\sim 5$ years by the remote handling manipulator. Based on evaluations using the plasma parameters on the divertor targets calculated with divertor simulation code SONIC [3][4], W with a thickness larger than $0.4 \mathrm{~mm}$ is necessary at the divertor targets.

One of the reliable methods to realize the tungsten divertor target in JT-60SA is replacement of the CFC monoblock target by the $\mathrm{W}$ bulk monoblock target. However, the weight of the divertor cassette with the $\mathrm{W}$ bulk monoblock target is evaluated to be $\sim 1.0 \mathrm{t}$, which is higher than the weight limit of the remote handling manipulator $(\sim 0.8 \mathrm{t})$. Hence, for the first phase with metallic walls it is planned to apply W coating and/or W plating on the actively cooled CFC monoblock divertor target without significant design changes of the divertor cassette and the actively cooled CFC monoblock divertor target.

Tungsten coatings with a thickness of $0.2 \mathrm{~mm}$ with Re-W multi inter-layers were produced on CFCs by vacuum plasma spraying (VPS) to be employed as divertor PFCs in the JET tokamak. Steady-state thermal heat flux tests by an electron beam irradiation showed one of the failure modes that melting of Re-W alloy decreases thermal conductivity between $\mathrm{W}$ coatings and CFCs, and this leads to overheating and melting of the W coating [5]. In ASDEX, 
$0.2 \mathrm{~mm}$ thick W-coated FGG tiles were employed at the outer strike position. Delamination of the $\mathrm{W}$ coating was found at heat fluxes up to $15 \mathrm{MW} / \mathrm{m}^{2}$ [6].

Recently, $0.4 \mathrm{~mm}$ thick VPS W coatings without any interlayers have been newly developed on CFCs with similar dimension as the actively cooled monoblock CFC in JT-60SA [7][8]. In the present study, heat fluxes up to $15 \mathrm{MW} / \mathrm{m}^{2}$ which represent the maximum heat fluxes expected at the JT-60SA divertor target were applied to the W coating on CFCs under adiabatic conditions to investigate tolerable heat loads. Heat transfer analyses of the W-coated CFC samples showed that the actively cooled W-coated CFC monoblock divertor target is feasible in JT-60SA applying only a minor design change.

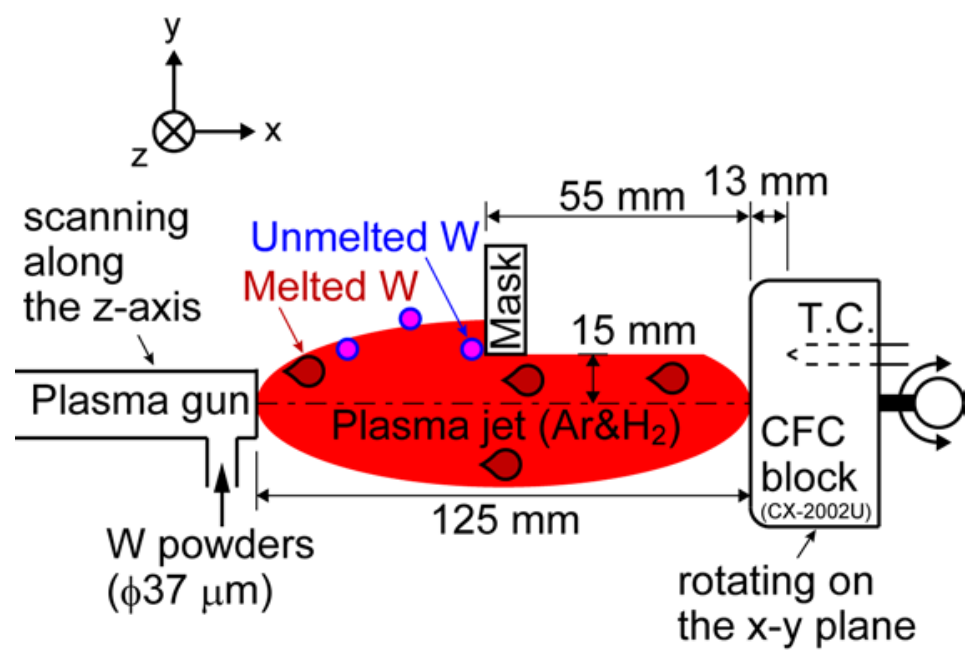

Figure 1. Schematic view of the VPS method applied in the present study. The plasma gun is scanned along the z-axis (vertical direction) and the CFC block is rotated on the $x-y$ plane (horizontal plane) to produce a uniform $W$ coating. $X$-, $y$ - and z-axis are inserted at the upper left.

\section{Experimental setup}

\subsection{Tungsten coating}

Tungsten with a thickness of $0.4 \mathrm{~mm}$ was coated by VPS on a CFC block (CX-2002U, Toyo Tanso Co. Ltd.) with a surface of $33 \times 31 \mathrm{~mm}^{2}$ and a thickness of $31 \mathrm{~mm}$. The surface area was comparable to that of the monoblock CFC $\left(32 \times 30 \mathrm{~mm}^{2}\right)$ for the actively cooled CFC monoblock divertor target in JT-60SA. Figure 1 shows schematic view of the VPS method applied in the present study. The CFC surface was blasted by aluminum powder to increase adhesion strength at the interface between W coatings and the CFC block. Then, the CFC block was set at $135 \mathrm{~mm}$ from the plasma gun nozzle, and the CFC block was rotated on the x-y plane 
(horizontal plane). A mixed hydrogen and argon plasma flame was produced with the plasma gun (F4VB, Sulzer Metco Co.), which was scanned along the z-axis (vertical direction). After the CFC temperature measured by a chromel-alumel (K-type) thermocouple reached $1000{ }^{\circ} \mathrm{C}$, W powder with an average size of $37 \mu \mathrm{m}$ was fed into the plasma gun. A carbon plate was set at $15 \mathrm{~mm}$ from the central axis of the plasma flame and at $55 \mathrm{~mm}$ from the CFC surface to prevent deposition of the unmelted W particles on the CFC surface (see Figure 1). Four CFC blocks could be coated in one batch by this method.

\subsection{High heat flux test}

High heat flux tests were performed on the W coating on the CFC block in GLADIS by high energy $\mathrm{H}$ ion beam irradiation [9]. Figure 2 shows the heat flux profile on the samples in GLADIS for a central power density of $15 \mathrm{MW} / \mathrm{m}^{2}$. Because the heat flux had a two dimensional Gaussian profile, two samples were bolted on a water-cooled $\mathrm{Cu}$ heat sink using a carbon sheet for better thermal contact in such a way that both samples were exposed to the maximum heat flux. A K-type thermocouple was inserted from the back side of the samples to $13 \mathrm{~mm}$ beneath the surface to measure the CFC temperature.

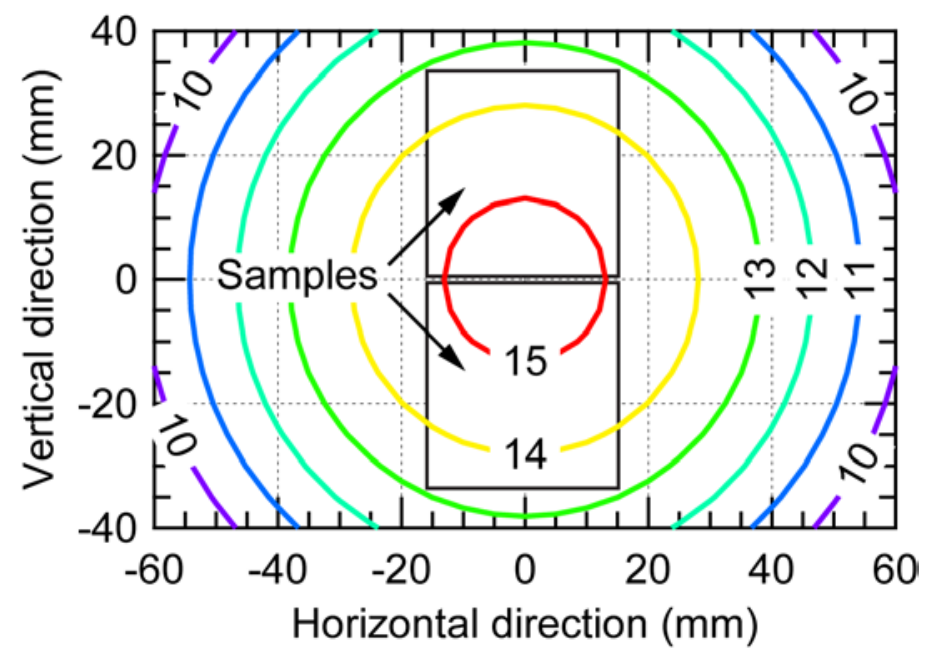

Figure 2. Heat flux profile on the samples in GLADIS for a central power density of $15 \mathrm{MW} / \mathrm{m}^{2}$.

Two kinds of high heat flux tests were performed: one is a screening test and the other is a cyclic test. The screening test was performed for two virgin samples to investigate tolerable heat loads. The screening test started at $6 \mathrm{MW} / \mathrm{m}^{2}$ for $2 \mathrm{~s}$. The heat flux and the pulse length were increased in a step-by-step manner up to $15 \mathrm{MW} / \mathrm{m}^{2}$ and $5 \mathrm{~s}$, respectively. It is noted that $15 \mathrm{MW} / \mathrm{m}^{2}$ corresponds to the maximum heat flux expected on the monoblock divertor target in 
JT-60SA. Cooling time was set to decrease to an initial temperature less than $25{ }^{\circ} \mathrm{C}$. Two or three pulses were applied at every step to detect damage productions on the $\mathrm{W}$ coating by temperature increase during the $2^{\text {nd }}$ and the $3^{\text {rd }}$ pulses. After the screening test, the cyclic test was performed to investigate the performance under cyclic loading for two samples: one is a virgin sample and the other is the screening-tested sample. Heat fluxes of $15 \mathrm{MW} / \mathrm{m}^{2}$ for $3 \mathrm{~s}$ were selected in such a way that the surface temperature reaches the one expected on the monoblock divertor target in JT-60SA, and the cycle number was 50. Additional 50 cycles at the same heat flux with increased pulse length of $4 \mathrm{~s}$ were applied. Surface modifications were monitored with a visible CCD camera, and surface temperatures at the center of the samples were measured with a one-color pyrometer using a typical emissivity of tungsten, 0.3 .

After the screening and the cyclic tests, secondary electron images and back scattering images were taken to investigate surface morphology and recrystallization, respectively, by a scanning electron microscope (SEM).

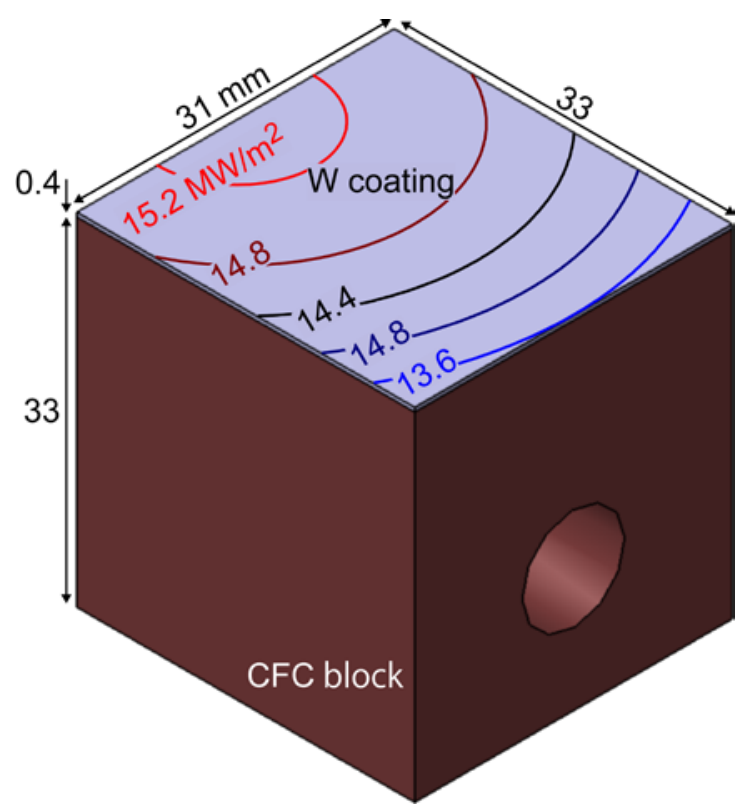

Figure 3. Analysis model of the W-coated CFC sample and heat flux profile for evaluation of thermal resistance.

\section{Heat-transfer analysis by finite element methods}

\subsection{Evaluation of thermal resistance between $W$ coating and a CFC block}

Heat transfer in the W-coated CFC sample was analyzed by finite element methods (FEM) using the ANSYS 18.0 software to evaluate thermal resistance between the $\mathrm{W}$ coating and the CFC block and heat transfer from the CFC block to the water-cooled $\mathrm{Cu}$ heat sink. 
Figure 3 shows the analysis model of the sample for evaluation of thermal resistance. Dimensions were the same as for the tested sample. Heat fluxes with a two dimensional Gaussian profile with a peak heat flux of $15.3 \mathrm{MW} / \mathrm{m}^{2}$ and FWHM of $78 \mathrm{~mm}$ were assumed on the W coating. Emissivity of the W coating and the CFC block was set to be 0.3 and 0.8 , respectively. The bottom surface of the sample was attached to the water-cooled $\mathrm{Cu}$ heat sink with $10^{\circ} \mathrm{C}$. Thermal resistance between the $\mathrm{W}$ coating and the CFC block was varied in order to match the measured and the simulated surface temperature of the $\mathrm{W}$ coating during the heating pulse. Heat transfer from the CFC block to the $\mathrm{Cu}$ heat sink was first fixed to $1 \mathrm{~kW} / \mathrm{m}^{2} \mathrm{~K}$ because heat transfer at the interface inserting carbon sheets was in the order of $\mathrm{kW} / \mathrm{m}^{2} \mathrm{~K}$ [10]. It is noted that the heat transfer is insensitive to the surface temperature for the short pulse injection where the heat flux to the $\mathrm{Cu}$ heat sink is not steady state. Then, heat transfer from the CFC block to the $\mathrm{Cu}$ heat sink was varied in order to match the measured and the simulated temperature of the CFC block during the cooling phase. The evaluated thermal resistance was applied between the W coating and the CFC block.

\subsection{Surface temperature of a $W$ coating on the CFC monoblock target}

The surface temperature of the $\mathrm{W}$ coating on the actively cooled CFC monoblock divertor target was evaluated by steady-state heat transfer analysis. Figure 4 shows the analysis model of the actively cooled W-coated monoblock CFC with the heat flux profile expected on the outer divertor target in JT-60SA. The thickness of the W coating is assumed to be $1 \mathrm{~mm}$. The dimensions of the W-coated monoblock CFC are identical to those of the monoblock CFC planned for JT-60SA. A copper alloy (CuCrZr) cooling tube with inner and outer diameters of $12 \mathrm{~mm}$ and 15mm, respectively, was brazed without any defects to the monoblock CFC with an oxygen free copper (OFCu) interlayer with a thickness of $1 \mathrm{~mm}$. the armor thickness, which is given by the distance between a plasma facing surface and an outer surface of the OFCu interlayer, is $8.5 \mathrm{~mm}$. A twisted tape was inserted into the cooling tube to improve heat transfer from the cooling tube to cooling water. Thickness and diameter of the twisted tape were $2 \mathrm{~mm}$ and $12 \mathrm{~mm}$, respectively, and twist ratio defined by 180 degree twist pitch against the inner diameter of the cooling tube was 2 . Cooling water with a temperature of $40{ }^{\circ} \mathrm{C}$ and a pressure of $2 \mathrm{MPa}$ was introduced to the cooling tube with a flow rate of $0.8 \mathrm{~kg} / \mathrm{s}$. The thermal resistance between the $\mathrm{W}$ coating and the CFC which was evaluated by analysis of the high heat flux test is applied. The heat flux distribution was evaluated by the divertor simulation code SONIC, and the absolute heat flux was varied as shown in Fig. 4. A toroidally symmetric heat flux distribution as indicated by the shaded area in Fig. 4 was assumed. 


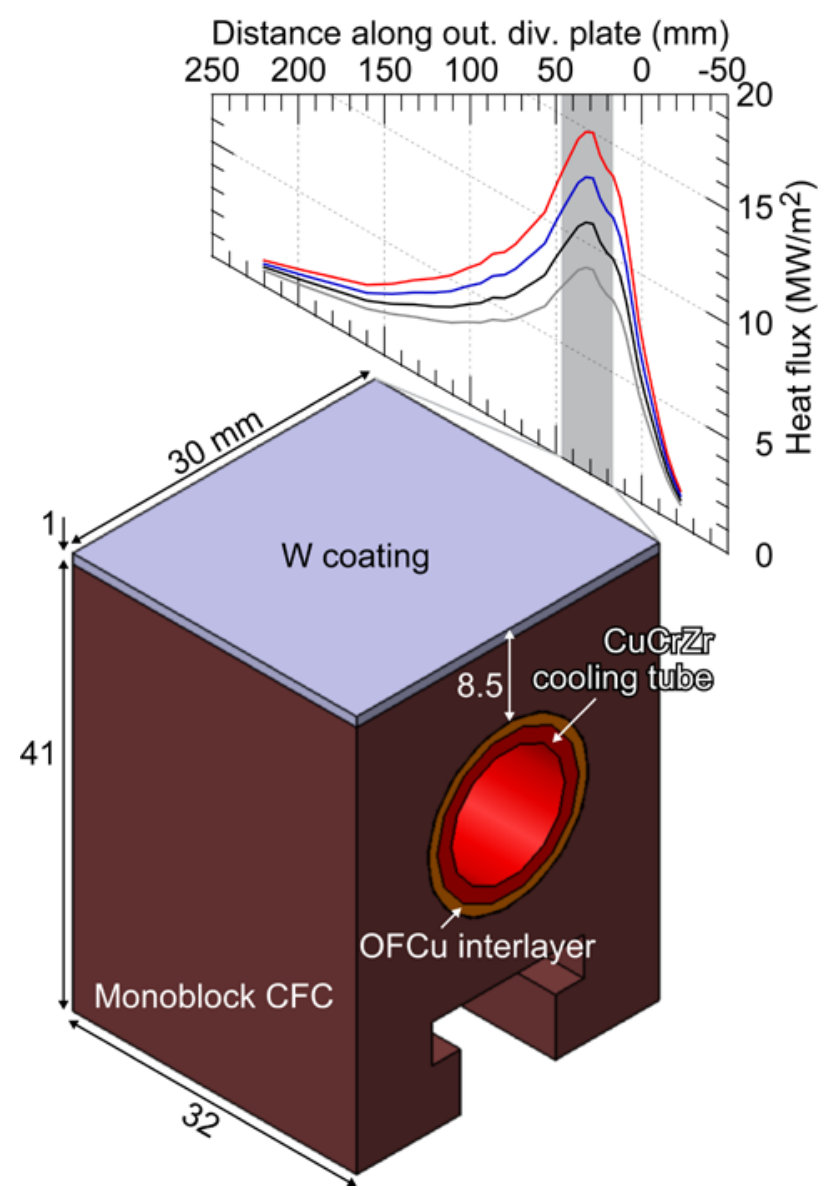

Figure 4. Analysis model of the actively cooled W-coated monoblock CFC and with the heat flux profile expected on the outer divertor target in JT-60SA. Armor thickness, distance between a plasma facing surface and an outer surface of the OFCu interlayer, is $8.5 \mathrm{~mm}$.

\section{Results}

\subsection{Screening tests}

Figure 5 shows the measured time evolution of surface and CFC temperatures for a heat flux of $15 \mathrm{MW} / \mathrm{m}^{2}$ for $5 \mathrm{~s}$ in the GLADIS facility. The initial temperature was $22{ }^{\circ} \mathrm{C}$. The surface temperature increased just after the heat flux was applied, and it reached $2000{ }^{\circ} \mathrm{C}$ at the end of the heat flux injection. Then, the surface temperature decreased to $580{ }^{\circ} \mathrm{C}$ at $27 \mathrm{~s}(22 \mathrm{~s}$ after switching off the beam). It is noted that the surface temperature with less than $400{ }^{\circ} \mathrm{C}$ cannot be measured by the one-color pyrometer used in the present study. The CFC temperature started to increase at $0.2 \mathrm{~s}$, and it reached its maximum of $870{ }^{\circ} \mathrm{C}$ at 8-9 s, 3-4 s after the end of the heat flux injection. Then, the CFC temperature decreased slowly to $630{ }^{\circ} \mathrm{C}$ at $27 \mathrm{~s}$. It is noted that the lower temperature at the surface at $27 \mathrm{~s}$ compared with that at the CFC would be lower emissivity of the $\mathrm{W}$ coating at low temperature. 


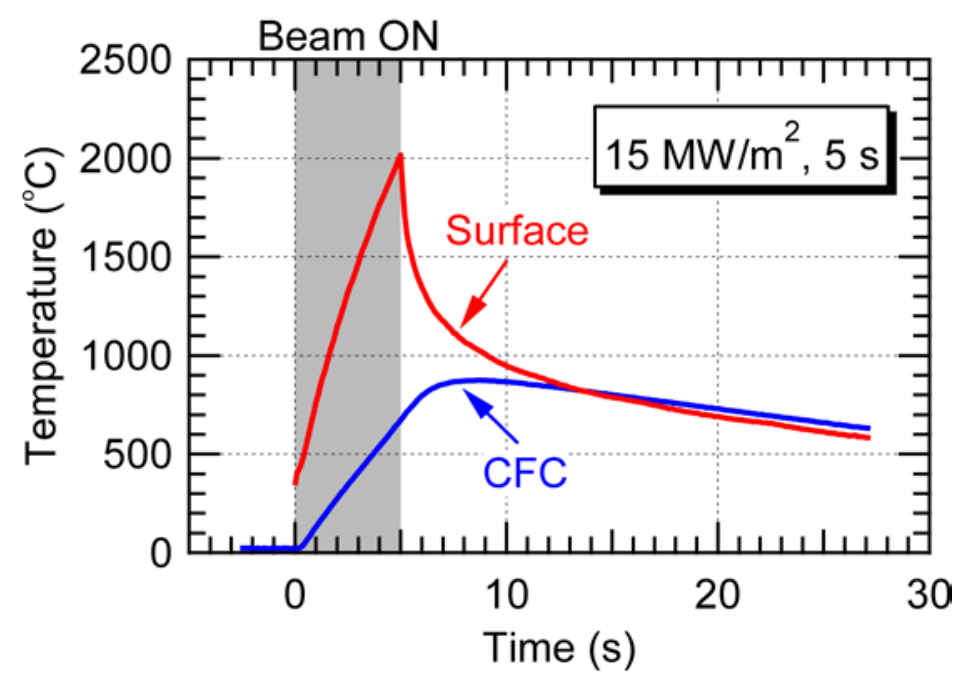

Figure 5. Measured time evolution of surface and CFC temperatures at $13 \mathrm{~mm}$ beneath the surface during and after the screening test for a heat flux of $15 \mathrm{MW} / \mathrm{m} 2$ for $5 \mathrm{~s}$.

Figure 6 shows the maximum surface temperature of the 0.4-mm thick $\mathrm{W}$ coating on CFCs at the end of the heat flux for different heat fluxes as a function of pulse length. The surface temperature increased with the pulse length and the heat flux density. No clear temperature increase was found at the $2^{\text {nd }}$ and the $3^{\text {rd }}$ pulses with the identical conditions. This result indicated that no severe damages reducing heat conductions such as delamination and exfoliation of the $\mathrm{W}$ coating were produced in the $\mathrm{W}$ coating. Similarly, no clear damages such as hot spots and droplets were observed by the visible CCD camera during the test.

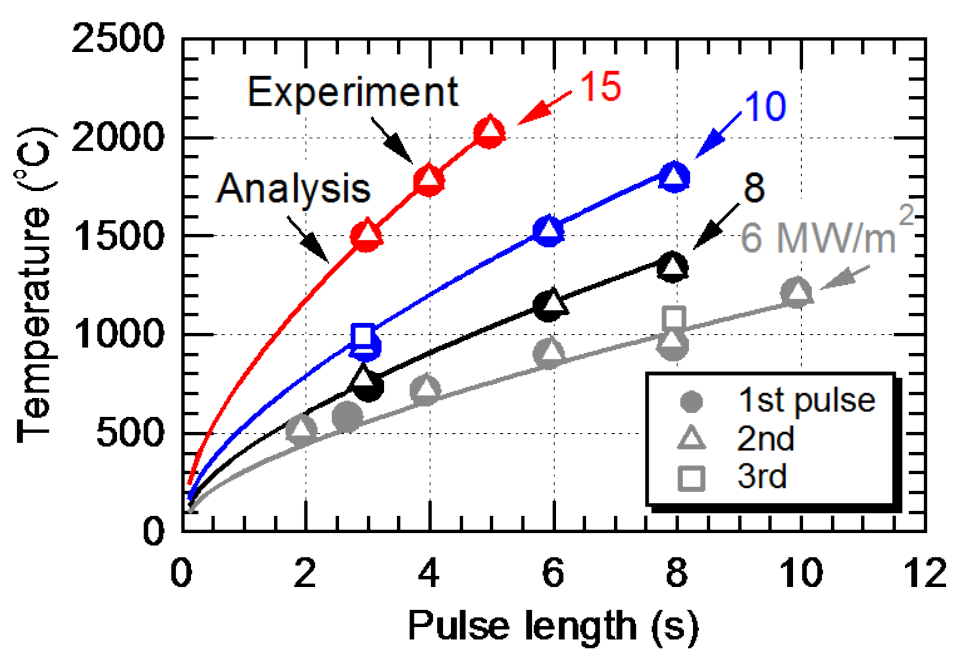

Figure 6. Maximum surface temperatures of the 0.4-mm thick W coating on CFCs at the end of the heat flux injection as a function of pulse length for different heat fluxes. Measured surface temperatures are shown by symbols and analyzed ones are shown by solid lines. Solid circles, open triangles and open squares indicate 1st, 2nd and 3rd pulses, respectively. 

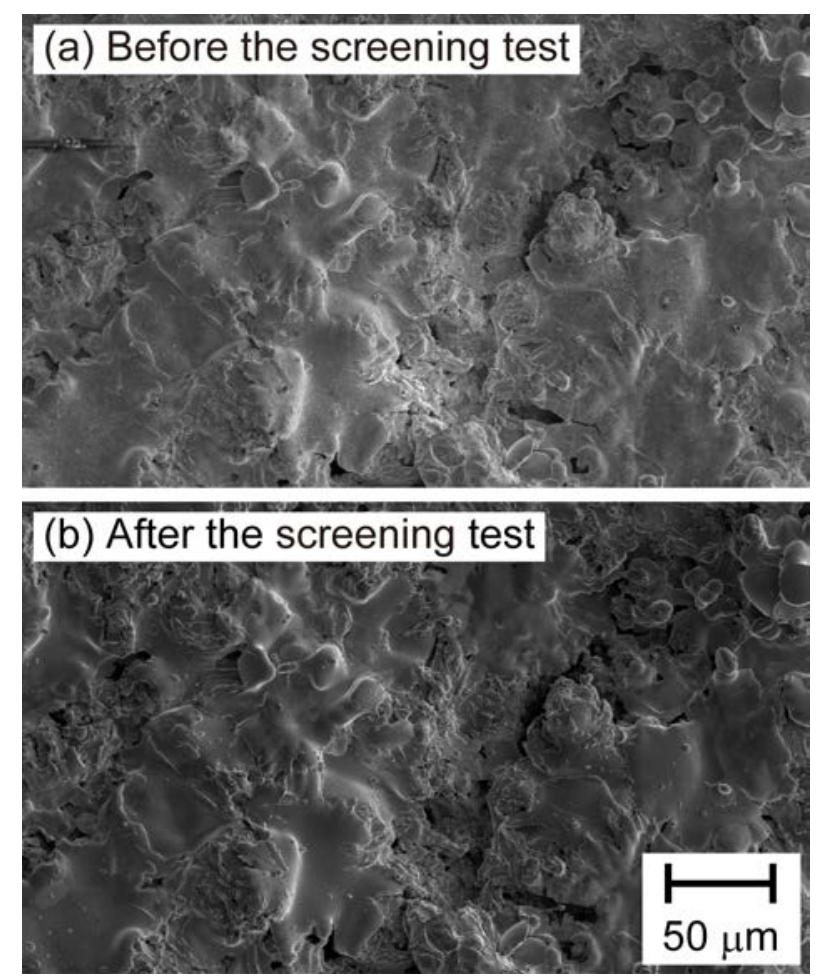

Figure 7. Secondary electron images of the W coating

(a) before and (b) after the screening test.

Figure 7 shows secondary electron images of the $\mathrm{W}$ coating (a) before and (b) after the screening test. No severe damages such as melting and clacking were observed on the $\mathrm{W}$ coating. However, recrystallization and grain growth were observed after the screening test, as shown in Figure 8. Small grains with a size of $\sim 10 \mu \mathrm{m}$ were formed in the VPS-coating process (as visible in Fig. 8 (a)), and these grains were recrystallized and grew up with a size of up to $\sim 40 \mu \mathrm{m}$ (as visible in Fig. 8 (b)). 

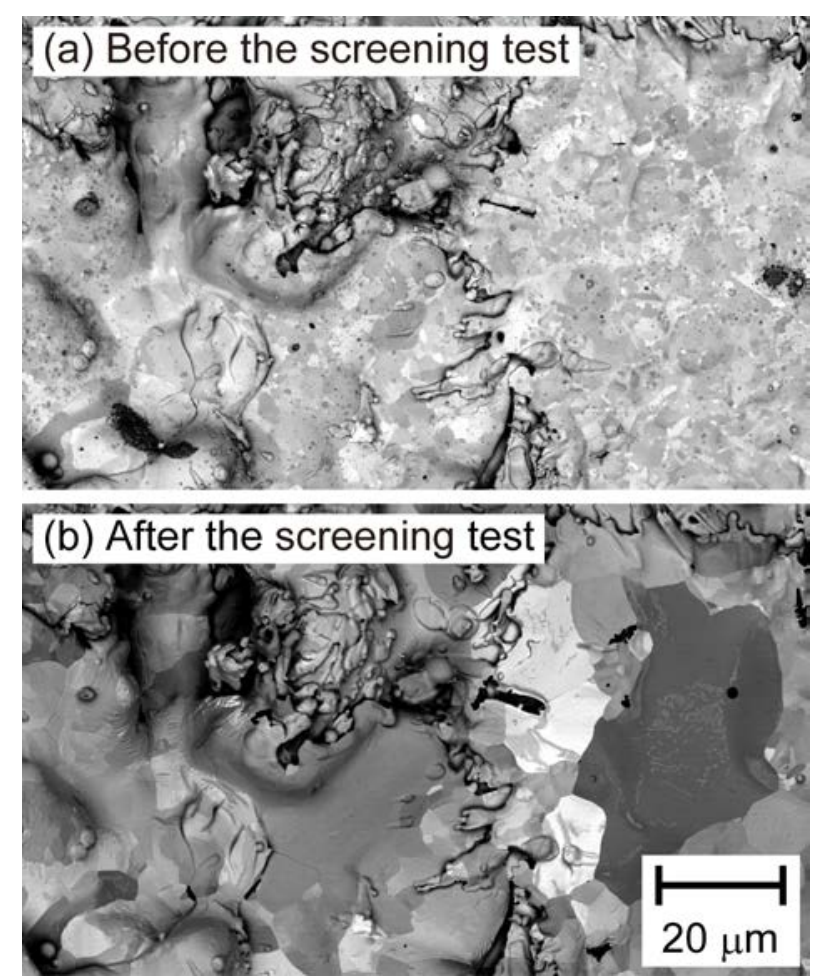

Figure 8. Backscattering electron images of the W coating (a) before and (b) after the screening test. Contrast shows different crystal grains.

\subsection{Cyclic tests}

Figure 9 shows the maximum surface temperature, the maximum CFC temperature and the initial temperature as a function of cycle numbers at $15 \mathrm{MW} / \mathrm{m}^{2}$ for $3 \mathrm{~s}$ and $4 \mathrm{~s}$. The initial temperature was kept below $70{ }^{\circ} \mathrm{C}$. The typical maximum surface temperatures were 1500 ${ }^{\circ} \mathrm{C}$ and $1770{ }^{\circ} \mathrm{C}$ for $3 \mathrm{~s}$ and $4 \mathrm{~s}$ loading, respectively, at the end of the heat flux injection. Neither a significant sudden increase nor a continuous increase of the surface temperature was observed within the 100 cycles. No significant changes of the CFC temperature and the initial temperature were also observed. These results indicated that no severe damages reducing the heat conduction such as delamination and exfoliation of the $\mathrm{W}$ coating were produced in the $\mathrm{W}$ coating. Similarly, no clear damages such as hot spots and droplets were observed by the visible CCD camera during the test.

Figure 10 shows secondary electron images of the W coating (a) before and (b) after the screening and the cyclic test. No severe damages such as melting and cracking were observed on the both-tested and the cyclic-tested (not shown) W coating. However, recrystallization and grain growth were observed after the cyclic test. 


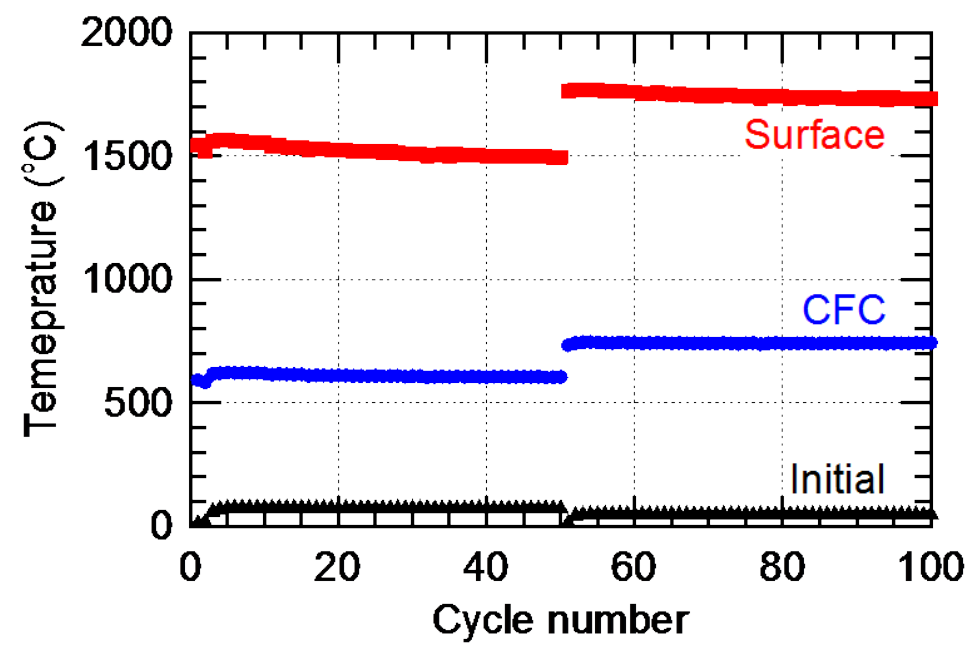

Figure 9. Maximum surface temperature, maximum CFC temperature and initial temperature at the end of the heat flux injection at $15 \mathrm{MW} / \mathrm{m} 2$ for $3 \mathrm{~s}$ and $4 \mathrm{~s}$.

The pulse length is increased to $4 \mathrm{~s}$ from the 51st cycle.
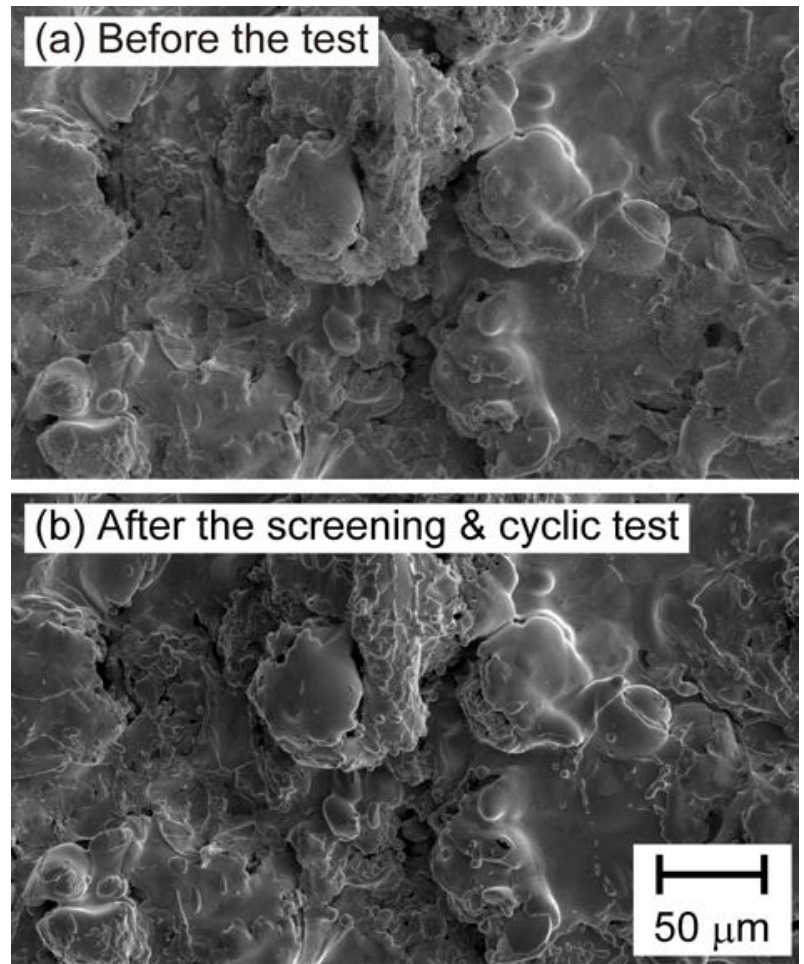

Figure 10. Secondary electron images of the W coating (a) before and (b) after the screening and cyclic test. 


\section{Discussion}

\subsection{Evaluation of thermal resistance between a W coating and a CFC block}

Two unknown parameters are evaluated by heat transfer analysis using FEM: one is thermal resistance between a W coating and a CFC block, the other is heat transfer from a CFC block to the water-cooled $\mathrm{Cu}$ heat sink. The evaluation of thermal resistance between a $\mathrm{W}$ coating and a CFC block is necessary for evaluation of surface temperature of a W coating on the actively cooled CFC monoblock divertor target in JT-60SA. Figure 11 (a) shows the time evolution of analyzed surface temperature with different thermal resistance at $15 \mathrm{MW} / \mathrm{m}^{2}$ for $5 \mathrm{~s}$. The analyzed surface temperature decreases with the thermal resistance, and it is consistent with the measured surface temperature in the case that the thermal resistance is $0 \mathrm{~m}^{2} \mathrm{~K} / \mathrm{W}$ or negligibly low during the heat flux injection phase. Then, heat transfer from a CFC block to the water-cooled $\mathrm{Cu}$ heat sink is varied in such a way that the analyzed CFC temperature is consistent with the experimental one at $0 \mathrm{~m}^{2} \mathrm{~K} / \mathrm{W}$ during the cooling phase. Figure 11 (b) shows the analyzed CFC temperature with different heat transfer at $15 \mathrm{MW} / \mathrm{m}^{2}$ for $5 \mathrm{~s}$.
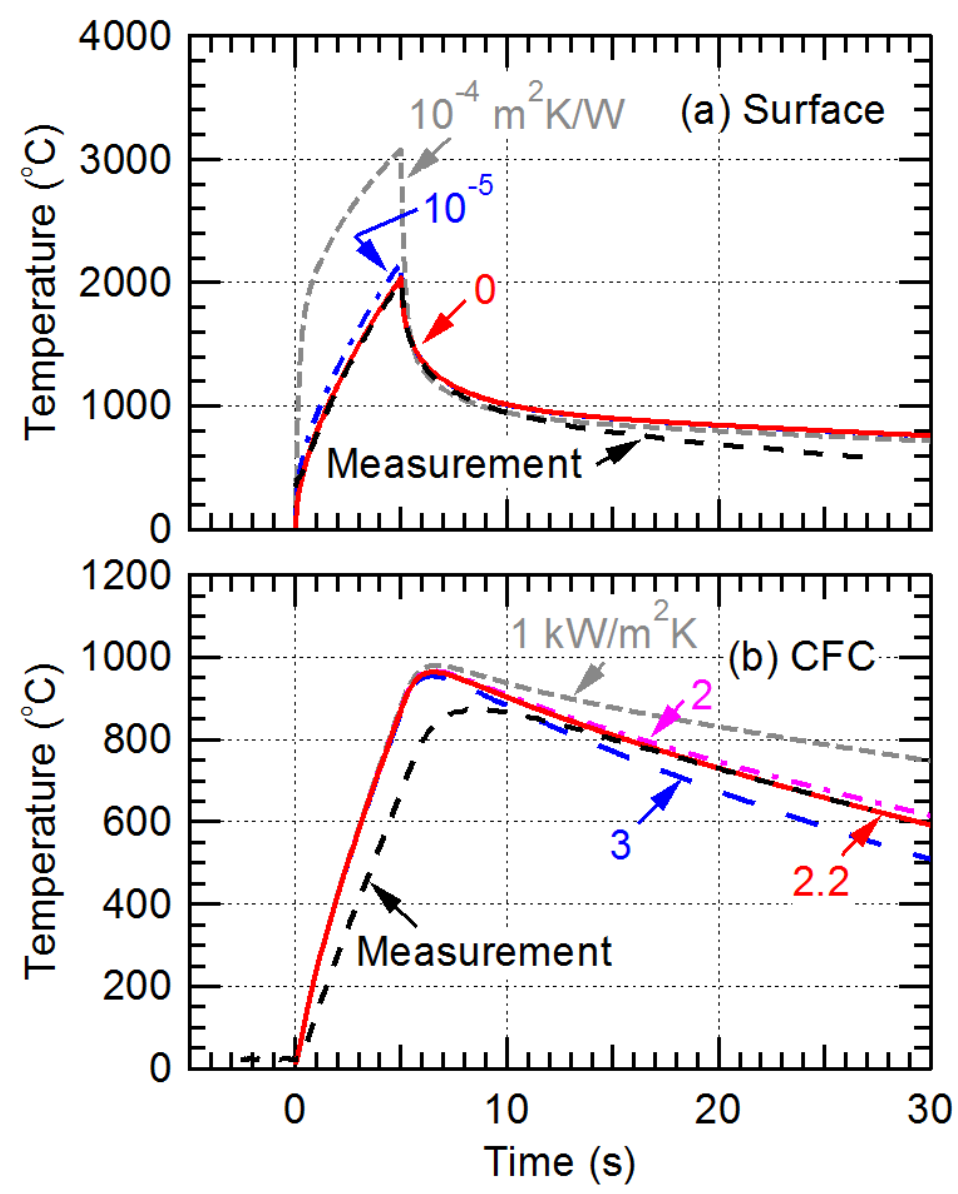

Figure 11. Time evolution of analyzed (a) surface temperature and (b) CFC 
temperature for different thermal resistance and heat transfer, respectively, at $15 \mathrm{MW} / \mathrm{m} 2$ for $5 \mathrm{~s}$. Measured surface temperature and CFC temperature are shown by broken lines for comparison.

The analyzed CFC temperature decreases with the heat transfer, and it is consistent with the measured CFC temperature during the cooling phase in the case that the heat transfer is 2.2 $\mathrm{kW} / \mathrm{m}^{2} \mathrm{~K}$. It is noted that the measured CFC temperature is lower than the analyzed one during the heat flux injection because the temperature increase is faster than the response speed of the thermocouple.

To confirm that the thermal resistance between a $\mathrm{W}$ coating and a CFC block is negligibly low and the heat transfer from a CFC block to the $\mathrm{Cu}$ heat sink is $2.2 \mathrm{~kW} / \mathrm{m}^{2} \mathrm{~K}$, heat-transfer analyses are also performed for the different heat flux conditions. The thermal resistance between a W coating and a CFC block is $0 \mathrm{~m}^{2} \mathrm{~K} / \mathrm{W}$, and the heat transfer from a CFC block to the $\mathrm{Cu}$ heat sink is $2.2 \mathrm{~kW} / \mathrm{m}^{2} \mathrm{~K}$. The analyzed surface temperature as a function of pulse length for different heat fluxes is shown in Fig. 6 by solid lines. The analyzed surface temperature is consistent with the measured surface temperature for all heat flux conditions with the identical parameters. This result indicates that the thermal resistance between a $\mathrm{W}$ coating and a CFC block is indeed negligibly low.

\subsection{Surface temperature of a W coating on the actively cooled CFC monoblock divertor target}

Surface temperature of a W coating on the actively cooled CFC monoblock target in JT-60SA is evaluated using the thermal resistance evaluated in the previous section. The maximum surface temperature of the $\mathrm{W}$ coating on the actively cooled CFC monoblock divertor target as a function of the peak heat flux is show in Fig. 12 by solid circles. The maximum surface temperature increases with the peak heat flux and it reaches to $1450{ }^{\circ} \mathrm{C}$ at $15 \mathrm{MW} / \mathrm{m}^{2}$ which is the maximum heat flux expected on the divertor target in JT-60SA. In the case of the original design the surface temperature is over the recrystallization temperature of tungsten, $1200{ }^{\circ} \mathrm{C}$. Once $\mathrm{W}$ is recrystallized, brittleness at grain boundaries is enhanced and fracture along grain boundaries easily occur, leading to grain injection into plasmas, and eventually to plasma disruptions in worst case. To avoid recrystallization of the $\mathrm{W}$ coating, the maximum heat flux onto the monoblock target should be reduced to less than $13 \mathrm{MW} / \mathrm{m}^{2}$.

One idea to decrease the maximum surface temperature less than the recrystallization temperature at $15 \mathrm{MW} / \mathrm{m}^{2}$ is reduction of the armor thickness, distance from a plasma facing surface to an outside surface of the OFCu interlayer, from the original design with $8.5 \mathrm{~mm}$. This should not negatively impact the lifetime since the erosion of $\mathrm{W}$ is calculated to be much lower than that of C. The surface temperature of the W coating on the actively cooled CFC monoblock 
divertor target with the reduced armor thickness with $4.5 \mathrm{~mm}$ (only the CFC thickness is decreased) is shown Fig. 12 by solid squares. Surface temperature of the W coating can be reduced to be $1100{ }^{\circ} \mathrm{C}$, clearly below the recrystallization temperature, at $15 \mathrm{MW} / \mathrm{m}^{2}$.

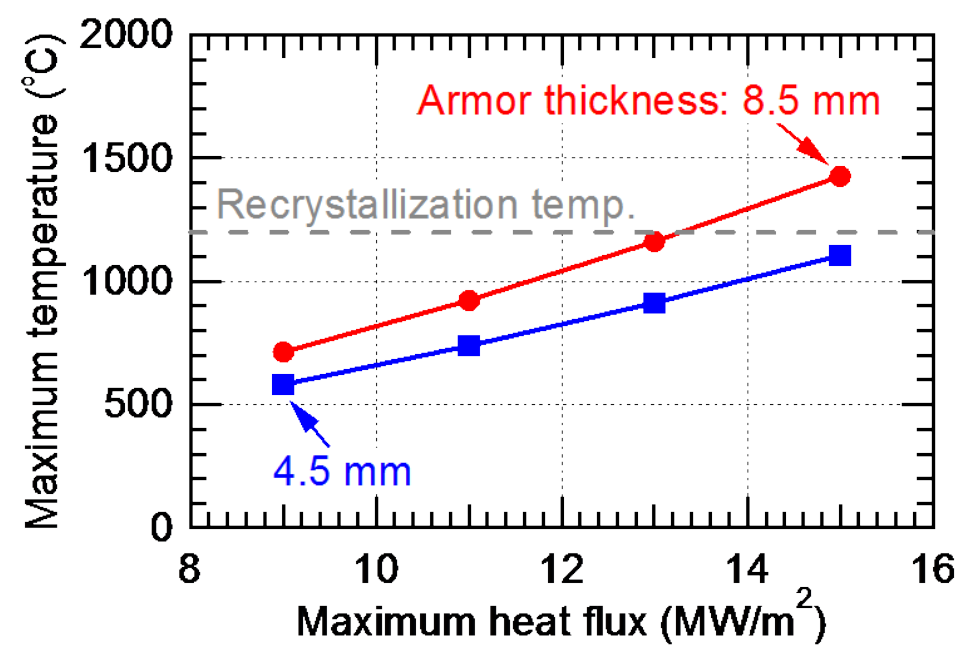

Figure 12. Maximum surface temperature of $\mathrm{W}$ coatings on the monoblack target in JT-60SA.

Circles show the surface temperature of the $\mathrm{W}$ coating with an armor thickness of $8.5 \mathrm{~mm}$ (original design). Solid squares show surface temperature of the $\mathrm{W}$ coating with the reduced armor thickness of $4.5 \mathrm{~mm}$. A broken line shows recrystallization temperature.

5.3. Further high heat flux test toward application to the divertor target in JT-60SA

From this work the $\mathrm{W}$ coating is found to withstand a heat flux of $15 \mathrm{MW} / \mathrm{m}^{2}$ which corresponds to the maximum heat flux expected on the monoblock divertor target in JT-60SA. On the other hand, about 3000 cycles of steady-state heat fluxes of $15 \mathrm{MW} / \mathrm{m}^{2}$ are expected on the $\mathrm{W}$ coating on the actively cooled CFC monoblock divertor target in JT-60SA within $\sim 5$ years. In addition, thicker $\mathrm{W}$ coatings up to $1 \mathrm{~mm}$ will be needed on the CFC monoblock divertor target in JT-60SA, compared to the thickness is $\sim 0.4 \mathrm{~mm}$ in this work. Thermal stress at the interface between $\mathrm{W}$ coatings and CFCs is expected to increase with the $\mathrm{W}$ thickness which increases the possibility of exfoliation of the $\mathrm{W}$ coating. Therefore, high heat flux tests are planned with thicker W coatings on the CFC monoblock divertor target.

\section{Conclusion}

High heat flux tests were performed on a $0.4 \mathrm{~mm}$ thick W coating on a CFC block (CX-2002U) produced by VPS for application to the future actively cooled W-coated CFC monoblock divertor target in JT-60SA. During the screening tests of up to $15 \mathrm{MW} / \mathrm{m}^{2}$ for $5 \mathrm{~s}$ of 
two virgin samples showed no severe damages, as for example melting, cracking, delamination and exfoliation on the $\mathrm{W}$ coating. However, the $\mathrm{W}$ coating was recrystallized, and the grain size increased from $\sim 10 \mu \mathrm{m}$ to $\sim 40 \mu \mathrm{m}$. Cyclic tests with 50 cycles at $15 \mathrm{MW} / \mathrm{m}^{2}$ for $3 \mathrm{~s}$ and additional loading with a longer pulse length of $4 \mathrm{~s}$ for a virgin and the screening-tested sample also showed no severe damages. However, recrystallization and grain growth were observed. Heat transfer analysis showed that thermal resistance between a W coating and a CFC block was negligibly low. This was probably because deposition of unmelted $\mathrm{W}$ particles was prevented by a mask, and adhesion of $\mathrm{W}$ coating was increased by blast treatment. The maximum surface temperature of a 1-mm thick $\mathrm{W}$ coating on the actively cooled CFC monoblock divertor target with the original design in JT-60SA would be above the recrystallization temperature of $1200{ }^{\circ} \mathrm{C}$, at a steady state heat flux at $15 \mathrm{MW} / \mathrm{m}^{2}$ which is the maximum heat flux expected on the divertor target in JT-60SA. Thinner armor thickness could be envisaged to prevent recrystallization of the $\mathrm{W}$ coating in the separatrix strike zone. High heat flux tests are planned to with thicker W coatings on the actively cooled CFC monoblock divertor target to demonstrate that steady-state heat fluxes of up to $15 \mathrm{MW} / \mathrm{m}^{2}$ can be removed without any damage such as exfoliation.

\section{References}

[1] JT-60SA Research Plan Ver. 3.32016 http://www-jt60.naka.qst.go.jp/jt60/pdf/JT-60SA\%20Research\%20Plan\%20Ver.3.3.pdf

[2] Sakurai S et al 2007 Fus. Eng. Des. 82 1767-1773.

[3] Kawashima H et al 2016 Contrib. Plasma Phys. 56 778-783.

[4] Hoshino K et al 2014 Contrib. Plasma Phys. 54 404-408.

[5] Hirai T et al 2009 J. Nucl. Mater. 392 40-44.

[6] Herrmann A et al 2009 Phys. Scr. T138 014059.

[7] Tokunaga T et al 2013 J. Nucl. Mater. 442 S287-91.

[8] Tokunaga T et al 2013 Plasma Fusion Res. 8, 1405111.

[9] Greuner H et al 2007 J. Nucl. Mater. 367-370 1444-1448.

[10] Herrmann A et al 2011 Phys. Scr. T145 014068. 